\title{
An Integrated Approach for Monitoring Service Level Parameters of Software-Defined Networking
}

\author{
Hui $\mathrm{Xu}$ and Hongwei Chen \\ School of Computer Science, Hubei University of Technology, Wuhan, China \\ xuhui@mail.hbut.edu.cn
}

\begin{abstract}
Software-Defined Networking (SDN) is a new-generation network that decouples the control plane and the data plane, and an additional management plane still lacks a mature implementation solution. In this case, monitoring service level parameters is of great significance to study issues related to service management for SDN. This paper then aims to apply the queuing theory and the extension theory to monitor service level parameters of SDN and propose an integrated approach based on both two theories from the formalization point of view. Simulation result and scenario analysis validate that, the proposed integrated approach prospects a feasible way of formalizing the solution to monitor service level parameters of SDN.
\end{abstract}

Keywords: Software-Defined Networking, service level parameters, queuing theory, extension theory, integration

\section{Introduction}

Compared to computer networking, Software-Defined Networking (SDN) tries to decouple the control plane and the data plane, and an additional management plane for SDN seems to be in need, but this management plane still lacks a mature implementation solution [1]. In this case, SDN services are provided by SDN service providers to meet the requirements of SDN service users, and as for SDN service users and providers, the specification of services in SDN needs to be seriously considered by terms of a targeted service level.

Service Level Agreement (SLA) is then introduced to define specific performance metrics and acceptable levels of network-provider performance with respect to these metrics [2]. SLA consists of service level objectives, and one main subject of service level objects is to monitor service level parameters. Similar to computer networking, service level parameters of SDN are used to describe the level of service provided by SDN service providers for SDN service users. Thus, monitoring service level parameters is of great importance to study issues related to service management for SDN. This paper then tries to propose an integrated approach based on both the queuing theory and the extension theory, in order to promote the formalization on monitoring service level parameters of SDN.

The remainder of this paper is organized as follows. Section 2 considers the use of Management Information Base (MIB) for monitoring service level parameters of SDN. Section 3 then proposes an integrated approach based on both the queuing theory and the extension theory to promote the formalization on monitoring service level parameters of SDN. Section 4 provides simulation result and scenario analysis in order to validate the feasibility of proposed integrated approach for formalizing the solution to monitor service level parameters of SDN. Section 5 concludes this paper. 


\section{Management Information Base for Monitoring Service Level Parameters of SDN}

Open Networking Foundation (ONF) is an organization to promote SDN standardizations, and it proposes the OpenFlow management and configuration protocol (OF-CONFIG, the newest version is 1.2 up to now) [3] for SDN management. OF-CONFIG adopts NETCONF [4] as the transport protocol, and current version 1.2 of OF-CONFIG uses the companion YANG module to realize its data model.

As the new-generation management information specification language proposed by Internet Engineering Task Force (IETF), YANG [5] can be used to define MIB as the data model for monitoring service level parameters of SDN. Figure 1 shows an example of management information definition using YANG from ietf-interfaces MIB as specified in RFC 7223 [6].

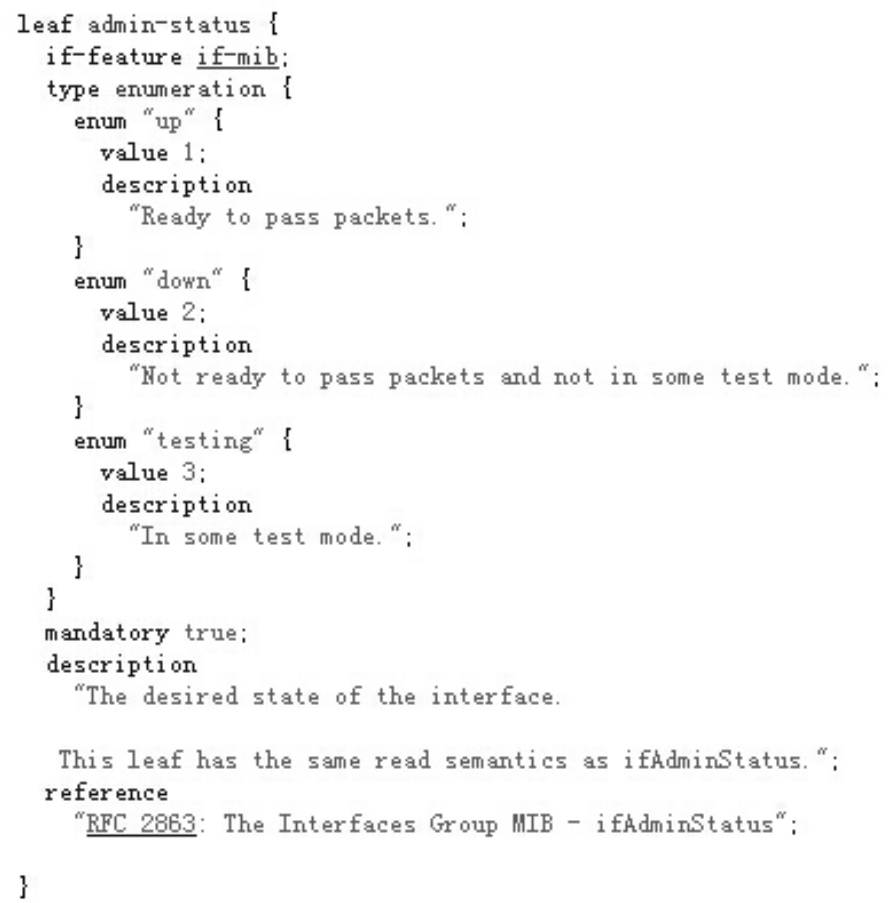

Figure 1. An Example of Management Information Definition from letfInterfaces Mib

As is indicated in Figure 1, when YANG is utilized to specify MIBs related to service level parameters of SDN, some lessons may be learned from existing management information definitions using Structure of Management Information version 2 (SMIv2), and in this example, the referred one is ifAdminStatus in the Interfaces Group MIB from RFC 2863.

\section{Proposed Integrated Approach for Monitoring Service Level Parameters of SDN}

As for monitoring service level parameters of SDN, the queuing theory is in favor of studying the regularity of the queuing model to monitor service level parameters of SDN. And based on this, the extension theory is integrated to prospect a promising solution to the quantitative change in this contradictory problem for 
monitoring service level parameters of SDN. An integrated approach based on both two theories above is then proposed from the formalization point of view.

\subsection{Application of the Queuing Theory}

When applying the queuing theory [7], the assumptions are that SDN assigns $m$ management processes independent of each other for job requests from SDN service users, and both job requests and management processes are supposed to be negative exponential distribution, expressed as Definition 1.

Definition 1 Assume that $\delta$ is a random variable and its probability density function is

$$
f(t)=\left\{\begin{array}{l}
\mu e^{-\mu t}, t \geq 0 \\
0, t<0
\end{array}(\mu>0)\right.
$$

, then it indicates that $\delta$ follows the negative exponential distribution, with its distribution function as

$$
F(t)=\left\{\begin{array}{l}
1-e^{-\mu t}, t \geq 0 \\
0, t<0
\end{array}(\mu>0)\right.
$$

If $\delta$ follows the negative exponential distribution as Definition 1, the mathematical expectation of $\delta$ is $\frac{1}{\mu}$, and the variance of $\delta$ is $\frac{1}{\mu^{2}}$.

As for the case of monitoring service level parameters of SDN, job requests from SDN users are supposed to be negative exponential distribution of parameter $\alpha$, and management processes from SDN service providers independent of each other are supposed to be negative exponential distribution of parameter $\beta$.

Thus from the viewpoint of the queuing theory, monitoring service level parameters of SDN can be seen as the $M / M / m / F C F S$ queuing model, in which $M$ means exponential distribution, $m$ is the assumed number of management processes independent of each other assigned by SDN for job requests from SDN service users, and FCFS indicates the First Come First Serve principle.

In this queuing model for monitoring service level parameters of SDN, $p_{x}=P\{X=x\}(x=0,1,2, \ldots)$ is introduced to define the possibility distribution for the number of SDN service users named as $X$. And since there are $m$ management processes assigned by SDN for job requests from SDN service users, then

$$
\begin{array}{r}
\alpha_{x}=\alpha, x=0,1,2, \ldots \\
\beta_{x}=\left\{\begin{array}{l}
x \beta, 1 \leq x \leq m-1 \\
m \beta, x \geq m
\end{array}\right.
\end{array}
$$

Suppose $\lambda=\frac{\alpha}{\beta}$ and $\lambda_{m}=\frac{\lambda}{m}$, when $\lambda_{m}<1$, according to the queuing theory, the possibility of idle state for $m$ management processes is

$$
p_{0}=\left\lceil\sum_{x=0}^{m-1} \frac{\lambda^{x}}{x !}+\frac{\lambda^{m}}{m !\left(1-\lambda_{m}\right)}\right\rfloor^{-1}
$$


Based on Formula (5), $p_{x}$ can be calculated as

$$
p_{x}=\left\{\begin{array}{l}
\frac{\lambda^{x}}{x !} p_{0}, 1 \leq x \leq m-1 \\
\frac{\lambda^{x}}{m ! m^{x-m}} p_{0}, x \geq m
\end{array}\right.
$$

As is indicated in Formula (6), when $x \geq m$, the next job request from SDN service users must wait, thus the Erlang waiting Formula then provides the possibility of the request waiting for management processes assigned by SDN as

$$
w(m, \lambda)=\frac{\lambda^{m}}{m !\left(1-\lambda_{m}\right)} p_{0}
$$

The average queue length for this queuing model to monitor service level parameters of SDN can then be defined as

$$
L_{q}=\frac{p_{0} \lambda^{m} \lambda_{m}}{m !\left(1-\lambda_{m}\right)^{2}}=\frac{w(m, \lambda) \lambda_{m}}{1-\lambda_{m}}
$$

Based on Formula (8), the average length for this queuing model to monitor service level parameters of SDN can be defined as

$$
L_{m}=\lambda+L_{q}
$$

According to Little Formula, the average waiting time $T_{w}$ and the average staying time $T_{s}$ can then be calculated as

$$
\begin{gathered}
T_{w}=\frac{L_{q}}{\alpha} \\
T_{s}=\frac{L_{m}}{\alpha}
\end{gathered}
$$

\subsection{Integration with the Extension Theory}

Based on the proposed queuing model for monitoring service level parameters of SDN, the quantitative change in this contradictory problem must be seriously considered from the viewpoint of formalization. Thus in this case, the extension theory extends the concept of distance to the concept of extension distance [8], and the extension distance uses the negative value to indicate the distance between the point in a section and the section itself. Definition 2 and Definition 3 respectively express the extension distance between a detected value from monitoring the service level parameter of SDN and one value domain or two value domains.

Definition 2 Assume that $V_{0}=\left\langle x_{0}, y_{0}\right\rangle$ is the acceptable value domain of a service level parameter of SDN in the real field, and $v$ is a detected value from monitoring this service level parameter, then the extension distance between $v$ and $V_{0}$ is defined as

$$
d\left(v, V_{0}\right)=\left|v-\frac{x_{0}+y_{0}}{2}\right|-\frac{y_{0}-x_{0}}{2}
$$


Definition 3 Assume that $V_{0}=\left\langle x_{0}, y_{0}\right\rangle$ and $V=\langle x, y\rangle$ are respectively the acceptable value domain and the complete value domain of a service level parameter of SDN in the real field, $V_{0} \subseteq V$ and $v$ is a detected value from monitoring this service level parameter, then the extension distance between $v$ and these two related value domains $V_{0}$ and $V$ is defined as

$$
D\left(v, V_{0}, V\right)=d(v, V)-d\left(v, V_{0}\right)
$$

Based on the extension distance formally specified by Definition 2 and Definition 3, the dependent function is then introduced by the extension theory to describe the degree of which things have a certain property according to historical data or professional standards [8]. As for the case of monitoring service level parameters of SDN, the dependent function is utilized to avoid subjective judgment, and with the use of the acceptable value domain and the complete value domain, the association degree can be confirmed by the dependent function, expressed as Definition 4.

Definition 4 Assume that $V_{0}=\left\langle x_{0}, y_{0}\right\rangle$ and $V=\langle x, y\rangle$ are respectively the acceptable value domain and the complete value domain of a service level parameter of SDN in the real field, and $v$ is a detected value from monitoring this service level parameter, then the dependent function between $v$ and these two related domains $V_{0}$ and $V$ is defined as

$$
a(v)=\left\{\begin{array}{l}
\frac{d\left(v, V_{0}\right)}{D\left(v, V_{0}, V\right)}, D\left(v, V_{0}, V\right) \neq 0, v \in V \\
-d\left(v, V_{0}\right)+1, D\left(v, V_{0}, V\right)=0, v \in V_{0} \\
0, D\left(v, V_{0}, V\right)=0, v \in V, v \notin V_{0}
\end{array}\right.
$$

As is indicated in Definition 4, the dependent function is utilized to implement the quantitative description of the association degree for the detected value from monitoring the specific service level parameter of SDN with its acceptable value domain and its complete value domain. If $a(v) \geq 0$, it means that the detected value satisfies the acceptable value domain of this service level parameter, and if $a(v)<0$, it reveals that the detected value is not related to the acceptable value domain of this service level parameter. However, when $-1<a(v)<0$, this unrelated situation can be improved by means of extension transformations based on the extension theory for the value of this service level parameter relating to its acceptable value domain.

\section{Validation}

In order to validate the feasibility of proposed integrated approach for promoting the formalization on monitoring service level parameters of SDN, this section first simulates a queuing model to monitor a specific service level parameter that is the waiting time, and then based on this, analyzes the corresponding scenario integrated with the extension theory. 


\subsection{Simulation Result}

As for proposed queuing model to monitor the validated service level parameter that is waiting time, Node no. 0 represents as an integrated management controller in SDN, and Node no. 1-10 are used for the purpose of distributed management in SDN. If at least one of Node no. 1-10 is changed in the service rate, it may play a great influence on monitoring the waiting time. Here, take Node no. 8 for example, and the number of jobs is set to 6000 . When the service rate for Node no. 8 varies from 0.1 to 1.0 , the waiting time for Node no. 0 will change accordingly, as demonstrated in Figure 2.

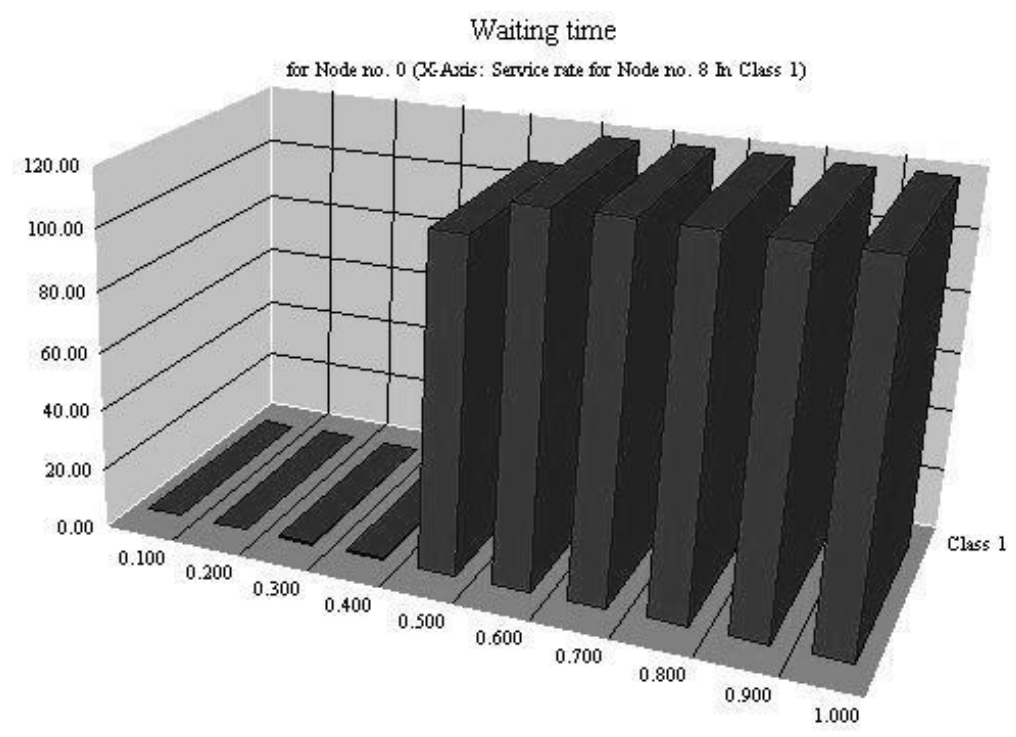

Figure 2. Waiting Time for Node No. 0 with the Changing Service Rate For Node No. 8

\subsection{Scenario Analysis}

Based on the simulation result above, this part discusses validation of proposed approach integrated with the extension theory. Consider the scenario of the waiting time for Node no. 0 above. Assume that, the acceptable value domain and the complete value domain of the waiting time for Node no. 0 are respectively $V_{0}=[0,40]$ and $V=[0,120]$, and current detected value of the waiting time for Node no. 0 from monitoring SDN is $v=60$.

a) According to Formula (12), $d\left(v, V_{0}\right)=\left|60-\frac{0+40}{2}\right|-\frac{40-0}{2}=20$ and $d(v, V)=\left|60-\frac{0+120}{2}\right|-\frac{120-0}{2}=-60$.

b) In this scenario $v \notin V_{0}$, then according to Formula (13), $D\left(v, V_{0}, V\right)=d(v, V)-d\left(v, V_{0}\right)=-60-20=-80$.

c) In this scenario $D\left(v, V_{0}, V\right) \neq 0, v \in V$, hence according to Formula (14), $a(v)=\frac{d\left(v, V_{0}\right)}{D\left(v, V_{0}, V\right)}=\frac{20}{-80}=-\frac{1}{4}<0$, which reveals that current detected value is not related to the acceptable value domain of the waiting time for Node no. 0. However, 
since $-1<a(v)=-\frac{1}{4}<0$, this unrelated situation can be improved by means of extension transformations based on the extension theory for the value of the waiting time for Node no. 0 relating to its acceptable value domain.

\section{Conclusions}

The main contribution of this paper is to study issues related to service management for SDN from the viewpoint of monitoring service level parameters, and to propose an integrated approach based on both the queuing theory and the extension theory, so as to promote the formalization on monitoring service level parameters of SDN. Simulation result and scenario analysis validate the feasibility of proposed integrated approach for formalizing the solution to monitor service level parameters of SDN.

\section{Acknowledgments}

This work has been supported by the Emergency Management Program for National Natural Science Foundation of China (No. 61440024), the Provincial Teaching Reform Research Project of Education Department of Hubei Province in China (No. 2012273), the Doctoral Scientific Research Fund from Hubei University of Technology (No. BSQD12029), the National Natural Science Foundation of China for Young Scholars (No. 61202287, No. 41301371) and the General Program for National Natural Science Foundation of China (No. 61170135). The authors would like to thank all project partners for their valuable contributions and feedbacks.

\section{References}

[1] S. Kuklinski and P. Chemouil, "Network Management Challenges in Software-Defined Networks", IEICE Transactions on Communications, vol. E97-B, no. 1, (2014), pp. 2-9.

[2] G. Huston, "Interconnection, Peering, and Settlements-Part I" The Internet Protocol Journal, vol. 2, no. 1, (1999), pp. 2-16.

[3] Open Networking Foundation, "OpenFlow Management and Configuration Protocol 1.2 (OF-CONFIG 1.2)", Available: www.opennetworking.org/technical-communities/areas/specification/1928-of-config, (2015).

[4] R. Enns, M. Bjorklund, J. Schoenwaelder and A. Bierman, eds, „Network Configuration Protocol (NETCONF)", RFC6241, (2011).

[5] M. Bjorklund ed, "YANG-A Data Modeling Language for the Network Configuration Protocol (NETCONF)", RFC6020, (2010).

[6] M. Bjorklund and A. Yang, "Data Model for Interface Management", RFC7223, (2014).

[7] D. Gross, J.F. Shortle, J.M. Thompson and C.M. Harris, "Fundamental of Queuing Theory (Fourth Edition)", Wiley Press, (2008).

[8] C. Y. Yang and W. Cai, "Recent Research Progress in Dependent Functions in Extension Sets", Journal of Guangdong University of Technology, vol. 29, no. 2, (2012), pp. 7-14.

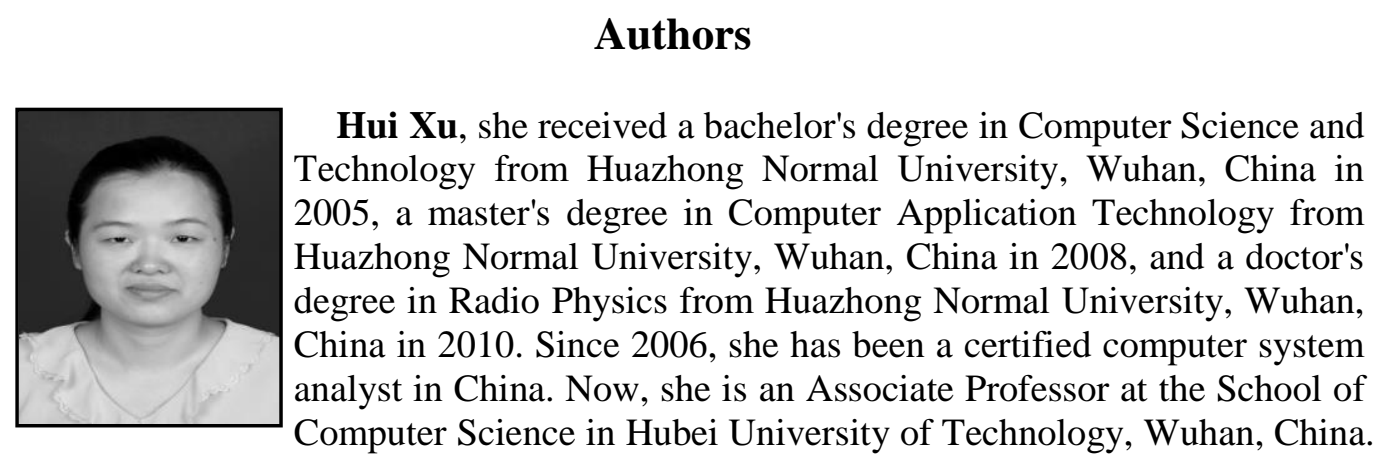


Currently, her major field of study is network and service management.

Dr. Xu became a Member of Institute of Electrical and Electronics Engineers (IEEE) in 2007, a Member of Association for Computing Machinery (ACM) in 2007 and a Member of China Computer Federation (CCF) in 2008. She has authored or coauthored 1 book and 2 book chapters in the field of network management, about 10 papers published by Chinese journals, more than 10 papers published by international journals, and more than 20 papers published by international conferences. In April 2008, she was awarded by International Association of Engineers (IAENG) for her firstauthored paper presented to 2008 IAENG International Conference on Communication Systems and Applications. Additionally, she was a Session Co-Chair or a Paper Reviewer for 2nd\&3rd\&7th\&8th International Conference on Computer Science and Education (ICCSE 2007\&2008\&2012\&2013), a Session Chair for 1st International Symposium on Electronic Commerce and Security (ISECS 2008), a Paper Reviewer for 4th IEEE Conference on Industrial Electronics and Applications (ICIEA 2009), a Paper Reviewer for 3rd International Conference on Computer and Network Technology (ICCNT 2011), a Paper Reviewer for 32nd Chinese Control Conference (CCC 2013), and a Paper Reviewer for Security and Communication Networks, an international journal published by Wiley Press.

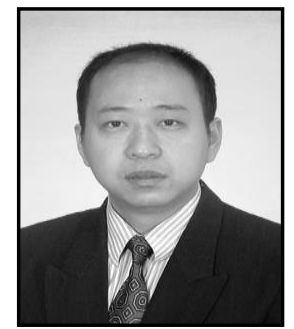

Hongwei Chen, he is a Professor at the School of Computer Science in Hubei University of Technology, Wuhan, China. Currently, his major field of study is distributed management. 